UCRL--91947

DE86 001559

\title{
CALCULATIONAL TOOLS FOR THE EVALUATION OF NUCLEAR CROSS-SECTION AND SPECTRA DATA
}

M. A. Gardner

This invited paper was prepared for submittal to the International Conference on Nuclear Data for Basic and Applied Science, Santa Fe, NM, May 13-17, 1985.

May 7,1985

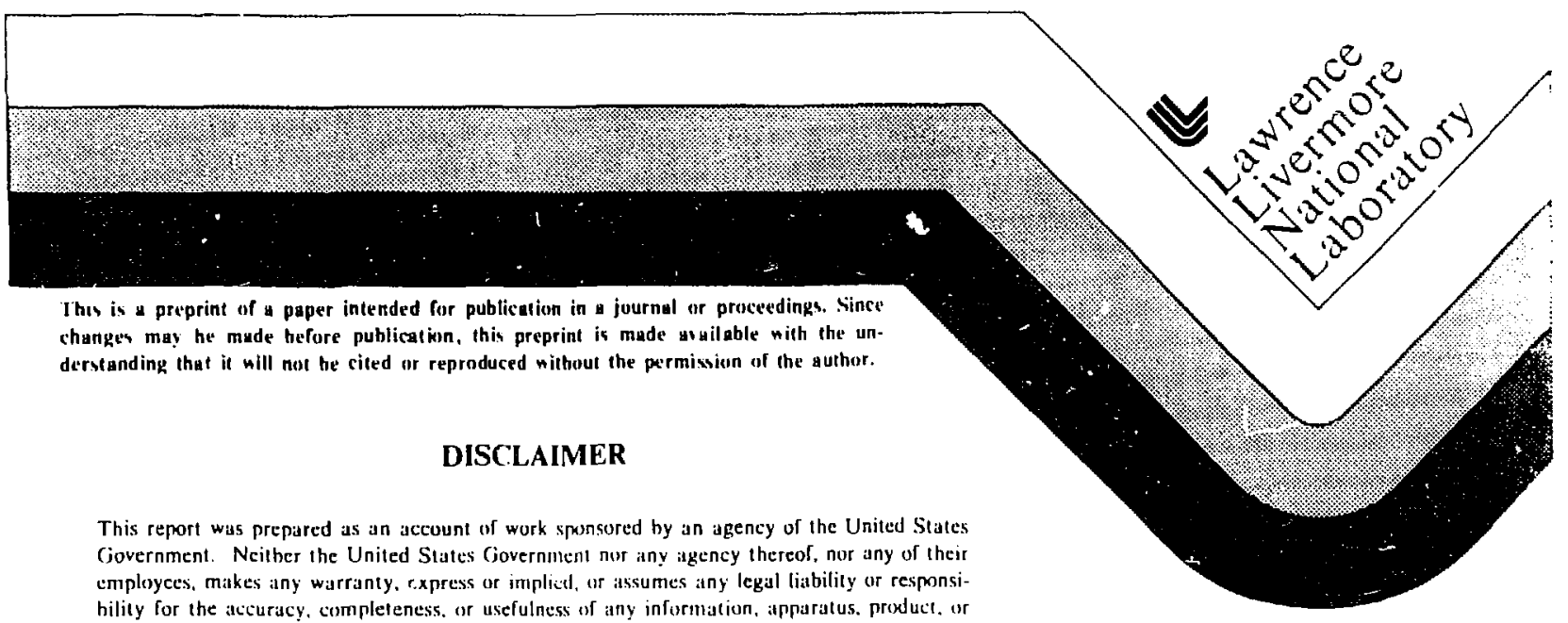
process disclased ar reprosents that its use would not infringe privately awned rights Refor ence herein to any specific commercial product, process, or service by trade name. trademark. manufinturer, or otherwise des not necessarily conslitute or unply its endersement, recommendiation, of favoring by the United Slattes firvernment or an! atgency thereof. The vitus and uninions of authors expressed herein do nol necessarily state or reflect those of the United States Ciovernment or any agency therees 
CALCLLATIONAL TOOLS FOR THE EVALUATION OF NUCLEAR CROSSSECTION AND SPECTRA DATA*

MAUREEN A. GARDNER

Lawrence Livermore National Laboratory, Livermore, CA, U.S.A.

Abstract continued improvements in the calculational methods for obtaining nuclear reaction cross sections and spectra provide indispensable tools for nuclear data evaluations.

\section{INTRODUCTION}

Significant progress has been made both in the development of model codes and in the methodology of obtaining the input parameters needed by them to allow today's evaluator to calculate reliable cross sections, isomer ratios, and gamma-ray and particle emission spectra. By using the various calculational tools now available, an evaluator confidently can determine such quantities in energy ranges where measurements have not been made, predict these quantities for unstable and excited-state targets and make choices between discrepant data sets. Throughout the nuclear data community there is growing recognition that calculational methods can and should be used in data evaluations as much as possible. This fact has been attested to in many presentations at this conference, as well as at other recent meetings such as the IAEA Third Advisory Group Meeting on Transactinium Isotope Nuclear Data, Uppsala, 1984'; the NEANDC Topical Conference on Measurements and Evaluations of Nuclear Data and Decay Heat for Fission Products, Tokai-mura, 1984,2 and the IAEA Consultants Meeting on Nuclear Data for Structurai Materials, Vienna, 1983.3 One of the main objectives at the Vienna meeting was the formulation of an IAEA-sponsored Coordinated Research Program on Methods for the Calculation of Fast Neutron Nuclear Data for Structural Materials. WhiTe the subject of my talk has many aspects that warrant attention, because of space limits, I will restrict my discussion here to recent developments in only a few areas of modeling and parameterizations associated with Hauser-Feshbach-type calculations.

* Work performed under the auspices of U.S. Dept. of Energy by Lawrence Livermore National Lab. under Contract No. W-7405-ENG-48.
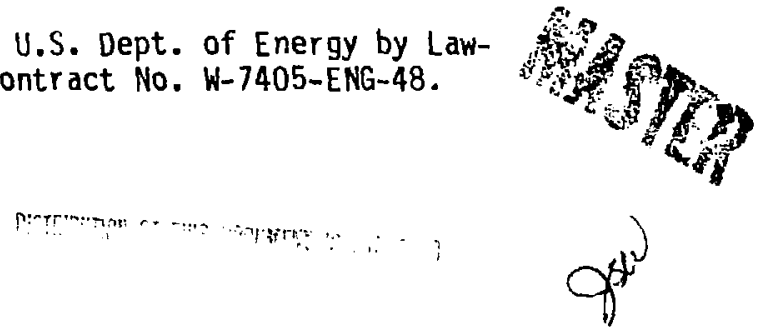


\section{A. GARDNER}

OPTICAL MODEL POTENTIAL (OMP) PARAMETERS

In making cross-section calculations, some of the first needs of an evaluator are appropriate sets of optical model parameters for the computation of the transmission coefficients for all particles involved, along with optical model codes that are easy to use and efficient, particularly in the coupied-channel case. Optical potentials should be suitable for several neighboring nuclei and must provide reliable transmission coefficients over wide energy ranges, from the highest incident energies down to a few keV. The latter requirement is, of course, very important for calculations near reaction thresholds; also, the examination of a typical neutron emission spectrum resulting from incident $14-\mathrm{MeV}$ neutrons, for example, shows that the production of a large number of fewMeV neutrons must be accounted for. In the past few years, evaluators have made extensive use of the Bruyeres-le-Chatel SPRT method 4 to obtain neutron and proton optical model parameters, because this appioach lends itself so well to meeting the above criteria for potentials. At the 1984 Conference on Neutron-Nucleus Collisions: A Probe of Nuclear Structure ${ }^{5}$ further improvements in OMP parameterizations were reported. Delaroche, et al., extended the SPRT method to include more observables and used a coupledchannel formalism to systematically study the energy dependence of the local neutron potential for deformed and vibrational nuclei using a large set of data measured over the range of $10 \mathrm{keV}$ to 80 MeV. Johnson described analyses that he and co-workers have been making of modern, high-resolution trarsmission measurements to deduce OMPs for individual partial waves, providing information beyond that available from s- and p-wave strength functions and the s-wave scattering racius. 7 These analyses should lead to improved understanding of potentials at low energies. Fig. 1, from Johnson's paper, shows, for 200-kev neutrons on $60_{\mathrm{Ni}}$, experimental compound and shape elastic cross sections (shaded regions) compared with those calculated with different s-wave imaginary and real well-depth values, WD and $V_{0}$, deduced using a coupledchannel analysis and a fixed geometry; solid points represent the accepted well denths. Several papers at the 1984 Conference reported on the satisfactory use of the semimicroscopic potential in studies

Figure 1 From the work of of Johnson, ${ }^{7}$ for ${ }^{60} \mathrm{Ni}$.

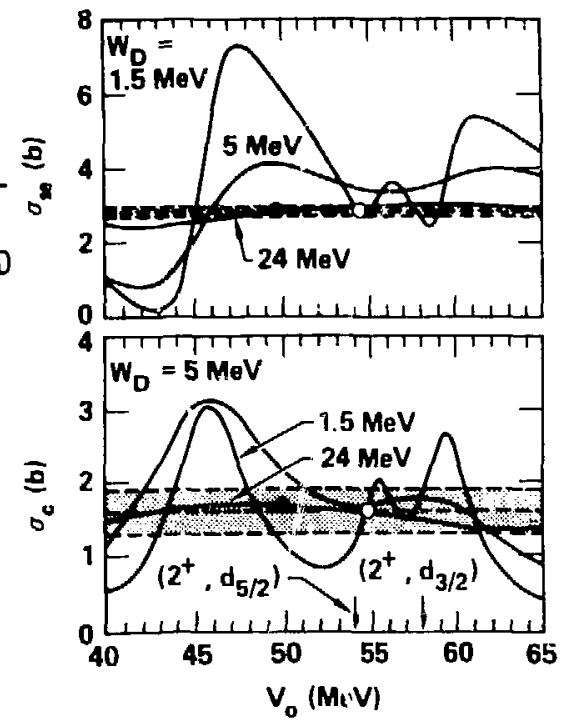




\section{CALCULATIONAL TOOLS}

on $208 \mathrm{pb}, 8239 \mathrm{pu}, 9$ and other actinides, 10 This model, with few adjustable parameters, promises to be a valuable predictive tool, providing a bridge between the more realistic representations of two-nucleon forces and the practicality of phenomenological models. On the subject of optical model codes, I would like to make two comments. The first is that we have found Bersillion's spherical OMP code, SCAT2,11 easy to use and accurate. The second is to point out that a recent coupled channel code study was sponsored by the NEA Data Bank. Comparisons were made of results obtained from CCROT/VIB, ADAPE, ECIS-79 and several versions of JUPITOR, and overall agreement was found to be quite good. Further details can be found in reports by Sartor 12 and Young. 13

\section{DISCRETE LEVELS AND LEVEL DENSITIES}

Good statistical model calculations require information about the discrete level structure and the level density as a function of energy, spin, and parity for each important nucleus. Uncertainties in the level density, especially as one moves away from the stability line or into regions of high excitation, typically can represent the largest contribution to the overall uncertainty in a calculated cross section. Fortunately, our knowledge in this area continues to improve; the Proceedings of the 1983 IAEA Group Meeting on Basic and Applied Problems of Nuclear Level Densities, 14 are recommended as a resource of information on the recent status of level density theory and model parameterizations. Papers were presented on a broad scope of subjects covering phenomenological, semi-microscopic, and microscopic Fermi-gas models and non-Fermigas approaches such as moment expansion, direct iterative, and number theoretical methods. Thorough reviews of the extraction of level density information from experimental data were also given, such as by Frohner 15 on the application of the tools of mathematical statistics to infer level densities from resolved resonances, and by Vonach 16 on deducing total level densities from particle emission spectra and other nonresonant cross-section data. General conclusions that can be reached from this 1983 Meeting, as well as from the recent literature, are as follows. Phenomenological level density models, such as the Gilbert-Camer on formalism, presently remain the easiest to use 17 and are adequate for most data evaluation calculations. 18 Microscopic Fermi-gas calculations of the Bardeen-Cooper-Schieffer-type (BCS), should be studied to see how better physics can be incorporated into the phenomenological model parameterizations. For example, BCS calculations can give information on the energy-dependence of the level-density parameter, "a,"19-21 and perhaps indirectly on the rotational enhancement observed at low excitation energies in deformed nuclei. It should be determined what is most furdamental to these kinds of calculations: the gap parameter, the pairing matrix element, the effect of blocking, how the sums over the levels should be made, what are 


\section{A. GARDNER}

the maximum $J$ values, etc. The other parameters should then be ferived, rather than used as additional input. In this way, BCS calculations can be used to make reliable predictions where no experimental information exists. Finally, as progress is made in calculations that include the full two-body force, either exactly or in approximate forms, more basic physics can be incorporated into the parameterizations of microscopic and phenomenological Fermi-gas models. Other speakers at the present Conference cover several of the above topics on level densities in detail; I now would like to discuss the ways in which discrete level information serves as an important tool in cross-section calculations and evaluations.

In our calculations at Livermore we continue to observe the necessity of describing nuclei in the first few MeV above their ground states with discrete levels. This discrete level information should not only consist of those levels that are obtained from experiment but must be supplemented with the level structure theoretically known to be present by model calculations. For aciurate cross-section and isomer-ratio calculations there appears to be no adequate way in which one can use a level density expression to represent or substitute for discrete levels. This is true both for spherical nuclei, where typically tens of levels are found in the first 1-4 Mev of nuclear excitation and for deformed nuclei, where perhaps a thousand levels are present in the first $1.5 \mathrm{MeV}$.

The importance of discrete level information is well known in the calculation of a cross section near its threshold. For example, when we calculated the $169 \mathrm{Tm}(n, 3 n)$ cross section for incident neutron energies in the first few MeV above the reaction threshold of $14.96 \mathrm{MeV}$, we used 63 modeled discrete levels for $167 \mathrm{Tm}$ in its first $1.4 \mathrm{MeV}$ of nuclear excjtation 22 and obtained agreement with the experimental data.23-25 When the cookmodified, 26 Gilbert-Cameron leve? density expression was used to describe 167 Tm down to its ground state, an excitation function was computed that was about $60 \%$ lower in the first $3 \mathrm{MeV}$ above the threshold.

Discrete levels are also essential to accurate calculations of neutron capture cross sections, as illustrated in two $89 y(n, y)$ excitation function calculations. 27 In the first 25 levels above the ground state were used to describe both the $89 y$ and $90 y$ nucle $i$ and, in the second, the levels were replaced with the cookmodified, Gilbert-Cameron level density formulae. While the first level in' $89 y$ lies $0.9 \mathrm{MeV}$ above the ground state and no inelastic scattering can occur until then, the calculation that used only level density expressions predicted an $\left(n, n^{\prime}\right)$ cross section that was already $400 \mathrm{mb}$ at $0.9 \mathrm{MeV}$. This, in turn, led to a significant lowering of the $(n, \gamma)$ cross section. A second, more surprisins: effect was observed in the keV energy range where the capture cross-section calculation using only level densities was still lower than that using 25 levels in $90 \%$. This was not due to inelastic scattering competition, but to the fact that all of the 


\section{CALCULATIONAL TOOLS}

partial radiation widths $(s, p, d$, etc.) were calculated to be about the same. When 25 levels were used in $90 \gamma$, the $p$-wave width was calculated to be twice as large as the s-wave width, in agreement with experiment, 28 and a larger capture cross section was obtained.

Discrete level sets then are not only of importance in the accurate description of nuclei at low excitation energies, but are also of importance in calculating valid radiation widths and in improving global level density parameterizations. It is now common for most statistical model codes to allow one to match the low energy portion of a level density expression with available discrete levels. Our experience has shown that this is valid in the case of spherical nuclei and that, if the level set is sufficiently conpiete, it can yield valuable information about the spin cutoff parameter as well as indicating what the other level density parameters should be to obtain good agreement with Dob at the neutron separation anergy and to obtain a reliable level density at higher energies as well.

In recent calculations that we made involving a series of $\mathrm{Sr}$ and $Y$ isotopes in the mass range of 86-90, almost every isotope required a different value of $k$, the constant in the mean square spin projection expression, $\left\langle m^{2}\right\rangle=k A^{2 / 3}$, and related to the energy-dependent spin cut-off parameter, ${ }^{2}(E)$, by the formula $\sigma^{2}(E)=\left(6 / \pi^{2}\right)\left\langle m^{2}\right\rangle(a U) 1 / 2$. While average values of $k=0.146$ or 0.24 have been recommended, 29,30 we found that the $k$ values, where we attempted to extract them from the discrete levels, ranged from 0.146 to 0.29 . Such fluctuations are in agreement with the observations of Reffo. 30 There are two ways in which $\sigma^{2}$ can be deduced from discrete level set.s. The first uses the maximum likel ihood estimator expression: $\sigma^{2}=(1 / 2 N) \sum_{j=1}^{N}(\mathrm{~J}+1 / 2)^{2}$, where there are $N$ levels of various $J_{j}$ spins and the second uses the expression: $P(J)=N(2 J+1)\left(2 \sigma^{2}\right)-1 \exp \left(-(J+1 / 2)^{2} / 2 \sigma^{2}\right)$. The first method can be more sensitive to small differences in $\sigma^{2}$ unless the levels exhibit a nonrepresentative distribution of mainly high or mainly low spins. Extracting $\sigma^{2}$ from the spin distribution, as given in the second expression, is useful unless several spins are missing. As shown in Finc. $2 a$ and $2 c$, difficulties are encountered for both $89 y$ (60 discrete levels) and $88 Y(25$ discrete levels) using the estimator method, since each nucleus has low-lying, high-spin states that introduce disproportionate weightings because of the $(\mathrm{J}+1 / 2)^{2}$ term. While the spin distribution method in Fig. $2 b$ indicates that $k=0.146$ is reasonable for $89 y$, the estimator method indicates that a slightly larger value of $k$ would be preferred. If one had information on the first 15 levels only, up to an excitation of $3.1 \mathrm{MeV}$, the choice of $k=$ 0.24 would be made. Choosing $k$ to be 0.24 instead of 0.146 alone, leads to about a $30 \%$ difference in the Fermi-gas level density. In the $88 y$ case, it is clear that the global $0.146 \mathrm{k}$ value is not suitable and $a k$ of about 0.29 is more appropriate, as verified by the spin distribution in Fig. 2d. We found that, in studying the 

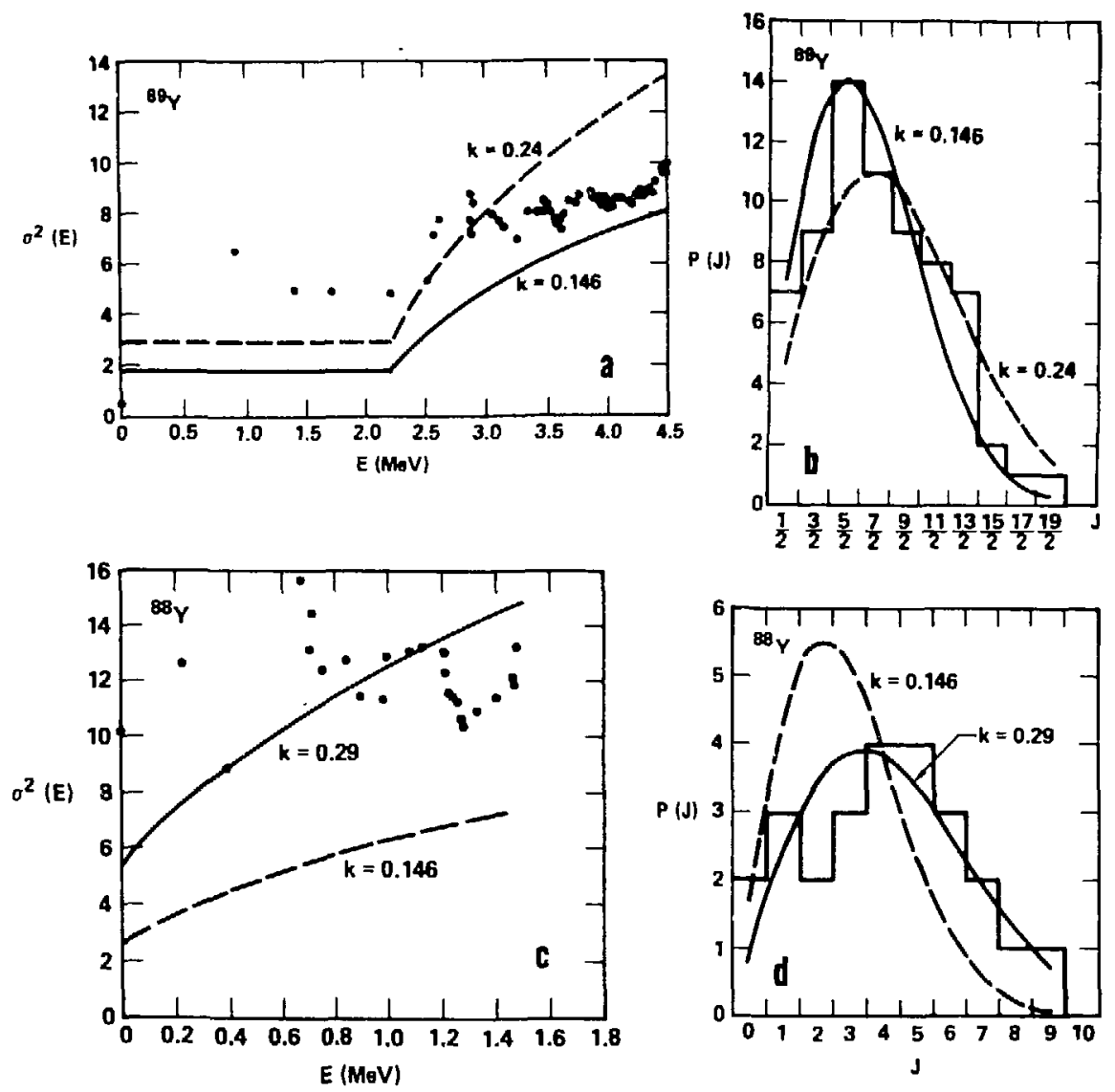

Figure 2a-d Spin cut-off information from discrete levels.

${ }^{89} Y(n, y){ }^{90} y$ cross section, the choice of $k=0.17$ instead of 0.146 , based on our ${ }{ }_{Y}$ discrete levels, led to a $D_{o b}$ change of $15 \%$ and a lowering of the capture cross section by about $10 \%$ in the $E_{n}$ range of a few hundred keV.

In the lanthanide and actinide regions, we have begun so use level structures modeled by Hoff, et al.31 For a deformed, oddodd nucleus, this method typically provides hundreds of discrete levels (sometimes up to a 1000) in the first $1.5 \mathrm{MeV}$ of nuclear excitation as well as gamma-ray branching ra+ios. The large number of discrete levels at low energies is expected due to rotational enhancement effects in these mass regions. It is not yet clear to us just how much level density parameter information can be extracted from these discrete levels, since the energy dependence of the rotational enhancement is still an open question. 32,33 


\section{CALCULATIONAL TOOLS}

However, we have made isomer-ratio çalculatjons for fjve $(n, y)$ and/or $(n, 2 n)$ reactions on $175 \mathrm{Lu}, 237 \mathrm{~Np}, 241_{\mathrm{Am}}$, and $243 \mathrm{Am}$, and have found that, in each case, the large modeled level set for each odd-odd product nucleus, along with gamma-ray branching ratios, was necessary to achieve agreement with experiment. All of the cross-section calculations were made with our version of the STAPRE code, 34 with no fission competition. Gamma-ray cascades leading to the ground-state and isomeric products were modeled using our $E I$ and $M I$ strength function systematics among the continuum bins while the discrete levels were depopulated according to the method of Ref. 31. For two reactions, $175_{L u}(n, \gamma)$ and $237 \mathrm{~Np}(n, 2 n)$, we tried using the same $E l$ and and $M I$ strength functions for both the continuum and among the discrete levels and obtained calculated isomer ratios equal to those obtained using the constant EI/MI ratio of 0.167 given in Ref. 31 .

The use of these modeled level sets in Hauser-Feshbach isomer ratio calculations promises to be a very useful tool for resolving differences in measured values and in predicting the energy dependency where measurements have not been made. For example, the $\mathrm{m} / \mathrm{g}$ ratio that we calculate for $175_{L u}(n, \gamma)$, using 291 modeled discrete levels for $176_{\text {Lu }}$ up to an excitation of $1.5 \mathrm{MeV}$ (including 62 rotational bands), is essentially energy independent for $E_{n}=0.01-0.3 \mathrm{MeV}$ and has a $30-\mathrm{keV}$ value of 1.6. This can be compared with two recent Maxwellian-averaged experimental values of 1.9435 and 3.55 .36 However, if, during the depopulation of the discrete levels, we allow E2 transitions to take piace
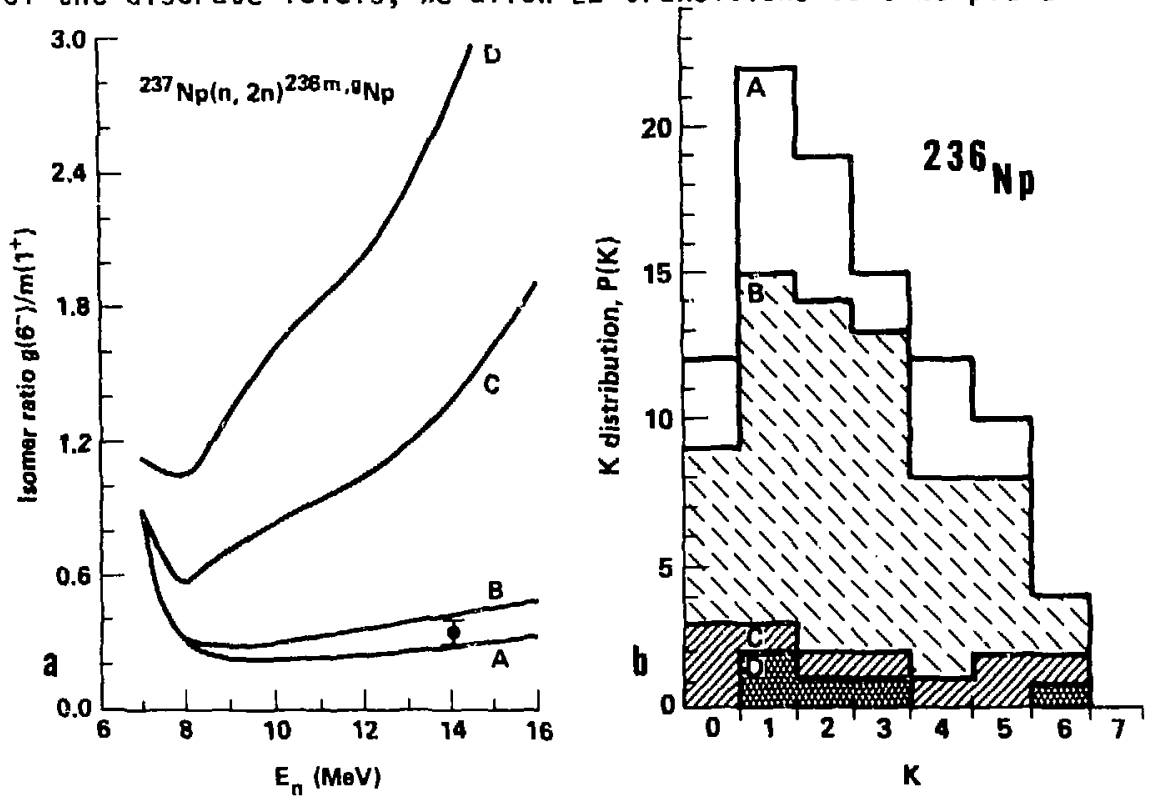

Figure $3 a, b \quad 237 \mathrm{~Np}(n, 2 n)$ isomer ratios and $P(K)$ for ${ }^{236} \mathrm{~Np}$. 


\section{A. GARDNER}

from some of the band heads, we compute a $30-\mathrm{keV} \mathrm{m} / \mathrm{g}$ ratio of 3.35. Further study of the importance of higher multipole transitions among the discrete levels in this kind of calculation is required before a conclusion can be drawn as to the preferred experimental value. In Fig. $3 a$, cur.ve $A$ shows our calculated $\mathrm{g} / \mathrm{m}$ ratio for $236 \mathrm{~Np}$ production via the $237 \mathrm{~Np}(n, 2 n)$ reaction from threshold up to $15 \mathrm{MeV}$. The short-lived, $22.5 \mathrm{~h}, 236_{\mathrm{Np}}$ (here assigned to be $236 \mathrm{~m}_{\mathrm{Np}}$ by the level modeling) beta decays $48 \%$ to $236 \mathrm{pu}$, which is of concern in reactor shielding. Curve $A$ was computed using 998 levels (including 94 rotational bands) to describe $236 \mathrm{~Np}$ in its first $1.48 \mathrm{MeV}$ of excitation; note that the experimental level information available for this nucleus consists of only 5 levels (including 3 rotational bands) up to $0.22 \mathrm{MeV}$. Curve $\mathrm{B}$ was computed with a $236 \mathrm{~Np}$ level set of 453 levels (71 rotational bands) obtained by truncating the 998-1evel set just below the 72nd band head. Both sets yield an isomer ratio in agreement with the single measured value at $14 \mathrm{MeV} .37$ Other such truncations led to curves $C$ (50 levels with 15 rot. bands) and $D(14$ levels with 5 bands). The distribution of $K$ quantum numbers, $P(K)$, for the level sets associated with curves A-D are given in Fig. $3 b$. Curves $C$ and $D$ were computed with level sets that do not have sufficiently representative samplings of $K$ values, leading to isomer ratios that are too high.

\section{GAMMA-RAY STRENGTH FUNCTIONS}

The magnitude and energy-dependence of gamma-ray transmission coefficients must be well characterized for accurate calculations of reutron-capture cross sections, gamma-ray production spectra, the competition between gamma-ray and particle emission near thresholds, isomer ratios, and photon-induced cross sections. In the past, the usual procedure was to normalize the magnitudes of the transmission coefficients to known ratios, $\left\langle\Gamma_{y}\right\rangle / D_{0 b}$, at the neutron separation energy, and where experimental values were unavailable, separate systematics for the prediction of $\left\langle\Gamma_{\gamma}\right\rangle$ or $D_{0 b}$ were evoked. We have found that the parameterization of the underlying quantity, the gamma-ray sirength function, was a more reliable way to predict gamma-ray transmission coefficients. 38 Recent$1 y$, we have derived absolute dipole strength function information for 176 Lu using the set of 291 modeled levels described above, and two types of experimental data: an average resonance capture (ARC) study of $175_{L u}$ using $2-k e V$ neutrons 39 and the neutron capture cross-section measurements of Macklin, et al. 40 and Beer, et al.41 for $E_{n}=30 \mathrm{keV}$ to $1 \mathrm{MeV}$. We found thax we needed to increase our previous estimate of the relative Mi/El strengths near $5 \mathrm{MeV}$ by a factor of 3, and to revise downward the absolute magnitude of our energy-dependent Briet-Wigner (EDBW) El strength function. By adjusting the one free parameter in our line shape we accomplished the latter, while still maintaining continuity with tine photonu- 


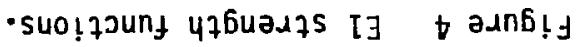

(n०W) ${ }^{l} \exists$ $\varepsilon t$

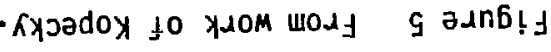

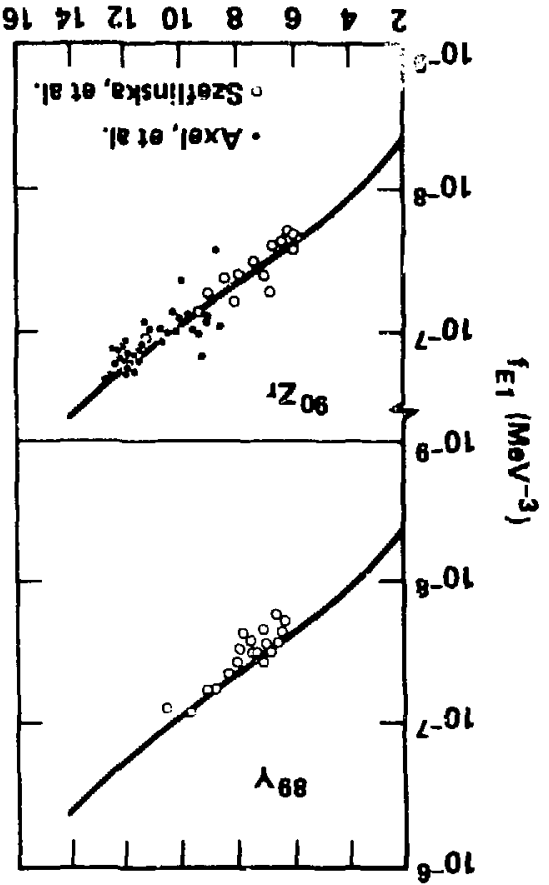

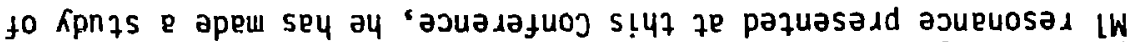
a47 to U0!7RE!7sasu! s!4 of Uo!f!ppe uI -suo!fount 476uadzs alod

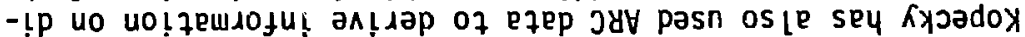

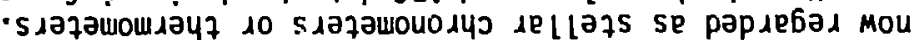

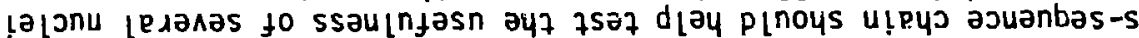

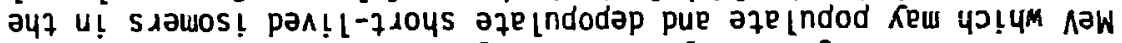

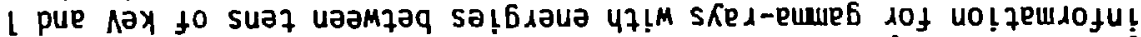

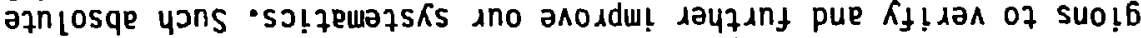

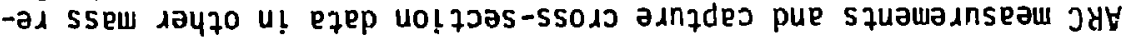

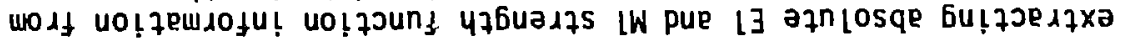

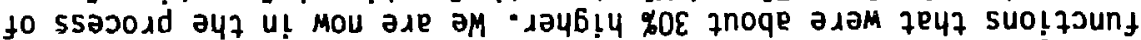

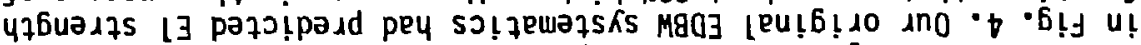

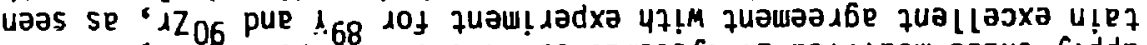

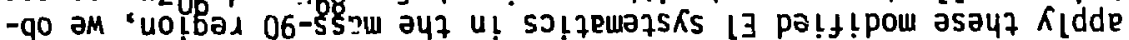

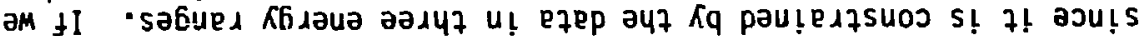

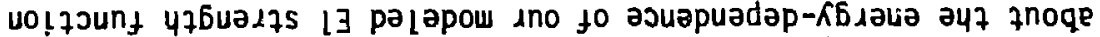

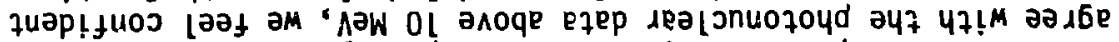

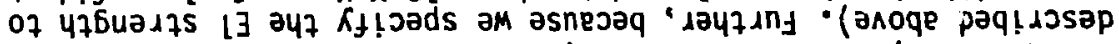
sflnsad 0 !fed dauros! ayz uo paseq) naw I punode pue (squawa inseaul

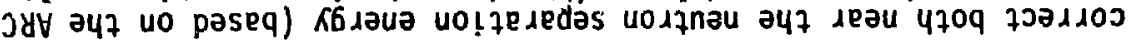
seadde mou suolfount 476uad7s LW pue 13 pajnpap asayl 'eqep deala 


\section{A. GARDNER}

the El/MI ratio over the entire mass range based on discrete resonance data 42 and more recently on ARC measurements. 43 As shown in Fig. 5, the ratios that he deduced from the ARC data (full circles) have less associated uncertainties than those derived from discrete resonance data (open symbols) and support the mass dependency that he previously had observed from the least squares fit to the discrete resonance ratios. He has also attempted to estimate MI/E2 ratios from ARC measurement.s.

Gardner has investigated

the relative importance of the modeling of the isoscalar E2 strengtb in the continuum for $=63 A^{-1} / 3$, and the peak width, $\Gamma_{\text {RE2 }}=18.9 \mathrm{~A}^{-1 / 3} \mathrm{MeV}$ were chosen, based on information about the isoscalar giant quadrupole resonance compiled by Bertrand. 45 A formula for peak crgss seption, $\sigma_{O E 2}=2.65 \times 10^{-2}\left(Z^{2} / \mathrm{A}^{2 / 3}\right) \mathrm{mb}$ was obtained by deriving an E2 sum rule from a prescription of Hayward 46 that related the magnitude of the E2 isoscalar quadrupole strength to the El dipole strength. This result is numerically similar to that deduced by Prestwich. 47 With these paral.eters, E2 strength functions were calculated using two line shapes: the Lorentzian and that of our EDBW El systematics. These results are shown in Fig. 6, as the shaded band, along with the newly deduced El and MI strength functions. The upper bound of the band results from the Lorentz line shape; the lower bound from that of the EDBW model. Note, in each case, that the E2 strength has been multiplied by $E^{2}{ }^{2}$ to provide an equal basis of comparison with the dipole strengths. One sees that over most of the energy range the E2 strength function is about two orders of magnitude lower than the dipole strengths.

Reffo has incorporated into his cascade model continuum $E l$, $M i$, and E2 strength functions based on Lorentz line shapes of the respective giant resonances; in additikn, ine supplements experimental branching ratios amony the discrete levels with estimated $E 1, M 1$, and E2 transitions where appropriate. The relative importance of such modeling is addressed by him in recent investigations of neutron capture cross-section and gamma-ray spectra calculations for $\mathrm{Nj}$ and $\mathrm{Fe}$ isotopes 48 and of isomer-ratio calculations on 24 Am. 49

Finally, I would like to point out another interesting calculational tool that should be useful in interpreting measured pri- 
CALCULATIONAL TOOLS

mary capture gamna-ray spectra. In Fig. 7 are shown calculated primary dipole transitions with energies in the range of 0.4 to $0.82 \mathrm{MeV}$, following $2-\mathrm{keV}$ neutron capture by $175 \mathrm{Lu}$ to the modeled 176Lu discrete levels described earlier. The positions of the levels accessible with dipole transitions and their spins and parities are indicated. The spectrum was constructed by smearing the calculated transition intensities with a unit gaussian line shape having a resolution of $6 \mathrm{keV}$. Some interesting features of the plot are: 1) for gamma-rays from 5.5 to $5.9 \mathrm{MeV}$ we see 5 doublets, 1 triplet and 1 quadruplet, all unresolved; more than expected on statistical grounds; 39 2) the $2^{-}+5^{-}$doublet just below $0.44 \mathrm{MeV}$ excitation appears to have the same shape and intensity as the singlet $4^{-}$peak at $0.466 \mathrm{MeV}$, while the triplet, quadruplet, and doubiet peaks at $0.66,0.75$, and $0.77 \mathrm{MeV}$, respectively, al so show a close resemblance in shape and intensity; and 3) the true singlet El peaks, due to transitions to $4^{-}$levels at $0.82,0.60$, and $0.47 \mathrm{MeV}$, show a pronounced increase in intensity, because of the energy dependence of the $E l$ strength function itself, in addition to the expected $E_{\gamma}{ }^{3}$ energy dependence.

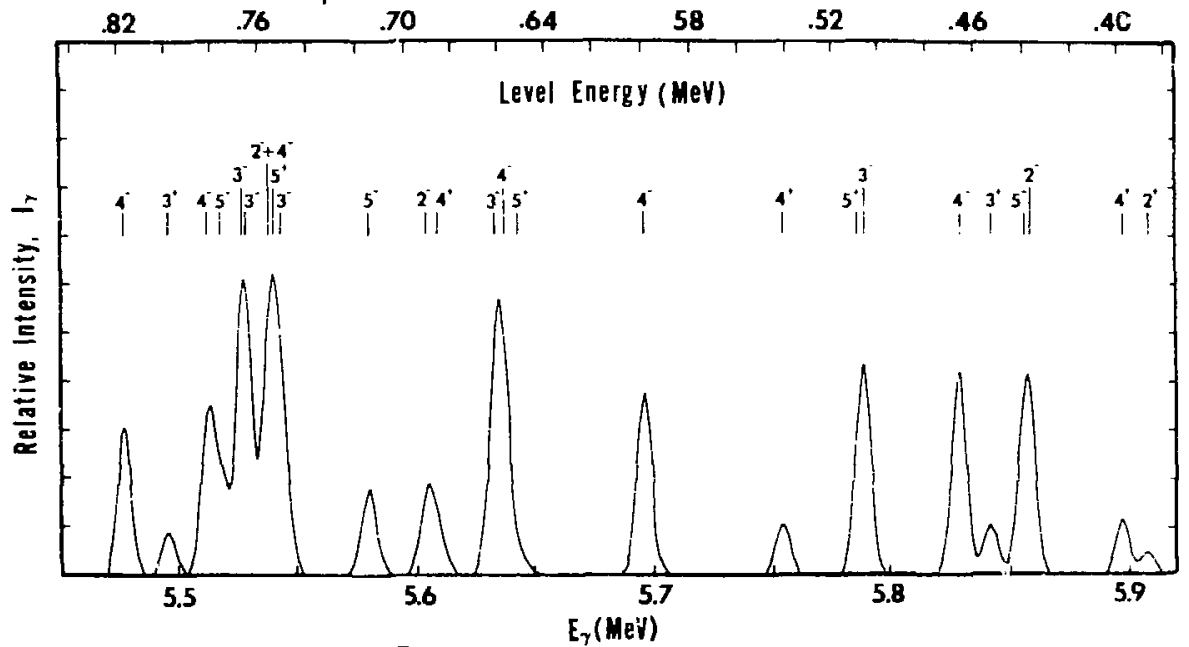

Figure 7 Calc. ${ }^{175}$ Lu(n,capture) primary gamma-ray spectrum. COVAR IANCE ANALYSES

One topic that has not received sufficient attention in the past is the application of variance-covariance analyses to nuclear model calculations. An excellent example of such work is that by Smith, et al.,50 in which covariances were determined for neutron cross sections calculated using a regional OMP parameterization based on optical model fits to experimental total and scattering cross sections for 11 elements, ranging from $Y$ to Sb. Also, at this Conference, the presentation of Uenohara, et al., describes a method of obtaining optimum level density parameters for several mass-90 nuclei using Bayesian statistics. 


\section{A. GARDNER}

\section{REFERENCES}

1. Summary Report of the Third Advisory Group Meeting on Transactinium Isotope Data, (Ippsala, 1984, ed. A. Lorenz, INDC(NDS)-158/L (1984).

2. Proc. NEANDC Conf. on Measurements and Evaluations of Nucl. Data and Decay Heat for Fiss. Prod.'s. Tokai-Mura, 1984, NEANDC(J) 100/U (1984).

3. Proc. IAEA Consultants' Meeting on Nuclear Data for Structural Materials, Vienna, 1983, ed. D. Cullen, INDC(NDS)-152/L (1984)

4. J. Delaroche, et al., IAEA-190 (1976) p. 251.

5.-10. Proc. Conf. on Neutron-Nucleus Collisions: A Probe of Nuclear Structure, Ohio, 1984, ed. J. Rapaport, et a!., AlP \# 124 (1985);

J. Delaroche, et al., p. 310; C. Johnson, p. 446; G. Haouat, et al.,

p. 320; C. Lagrange, et al., p. 318; L. Hansen, et al., p. 314

11. O. Bersillon, NEANDC(FR) $200<L>(1981)$.

12. E. Sartori, NEANDC-182 A (1984).

13. P. Young. "Status of Recent Nuclear Model Code Comparisons Organized by the NEA Data Bank." 24th Mtg. of NEANDC. 1984, NEANDC 195 "U"

14.-20. Proc. IAEA Advisory Grp. Mtg. On Basic and Applied Problems of Nuclear Level Densities, BNL, 1983, ed. M. Bhat, BNL-NCS-51694 (1983):

F. Frohner, p. 219; H. Vonach, p. 247; E. Arthur, p. 311; G. Reffo,

p. 203.; G. Mainu et al., p. 75; V. Ramamurthy, et al., p. 187.

21. A. Ignatyuk, et al., Sov. J. Nucl. Phys., 21, 255 (1975).

22. R. Hoff and W. Willis, UCAR $10062-84 / 1$ (1984) p. 3-33.

23. B. Bayturst, et al., Phys. Rev. C12, 451 (?975)

24. D. Nethaway, unpublished, (1972,1976); Nucl. Phys. A190, 635 (1972).

25. L. Veeser, et al., Phys. Rev. C16, 1792 (1977).

26. J. Cook, et al, AĀEC/TM-39? (1967).

27. D. Gardner, Neutron Radiative Cápture, (Pergamon, 1984), p. 62.

28. J. Boldeman, et al., Nucl. Sci. Eng. 64, 744 (1977).

29. A. Gilbert and A. Cameron, Can. J. Phys. 43, 1446 (1965).

30. G. Reffo, Moment Methods in Many-Fermion Systems,(Plenum, 1980), p. 167.

31. R. Hoff, et al., AlP \#125 (1985) p. 274; R Hoff, et al., UCAR 10062-83/1 (1983) p. 218; D. Gardner, et al., ibid. p. 51.

32. G. Hansen and A. Jenserl, BNL-NCS-51694 (1983) p. 161.

33. S. Kataria, et al., Phys. Pev. C18, 549 (1978).

34. M. Uhl, Acta Phys. Ausiriaca 31, 245 (1970).

35. H. Beer, et al., Phys. Rev. C30, 464 (1984).

36. B. Allen, et al., inst. of Phys. Conf. Series \# 62 (1982) p. 573.

37. W. Myers, et al., J. Inorg. Nucl. Chem. 37, 637 (1975).

38. D. Gardner, NEANDC(US)-214/L (1983) p. 67.

39. R. Hoff, et al., BNL-34811 (1984).

40. R. Macklin, et al., LA-7479-MS (1978).

41. H. Beer, et al., NBS Special Publ. 594 (1980) p. 340.

42. J. Kopecky, ECN Rpt. ECN-81-040 (1981).

43. J. Kopecky. AlP \# 125 (1985) p. 318.

44. D. Gardner, unpublished work (1984).

45. F. Bertrand, Ann. Rev. Nucl. Sci. 26, 457 (1976)

46. E. Hayward, Pholonuclear Reactions I, (Springer-Verlag, 1977), p. 340.

47. W. Prestwich, et al., Atoms and Nuclei 315, 103 (1984).

48. G. Reffo, INDC(NDS)-152/L (1984) p. 83 .

49. K. Wisshak, et al., Nucl. Sci. Eng. 81. 396 (1982)

50. D. Smith and P. Guenther, ANL/NDM-81 (1983). 